\title{
Paradigm Shift: Role of Social Media and Information Technology in Healthcare Advancements
}

\section{Ritesh Kumar*}

Department of Bioinformatics, University of Kansas, KS, USA

Health and Medical informatics is the intersection of information science, medicine and healthcare. It began to take-off in the United States in the 1950s with the rise of the microchip and computers. Over the course of time, health and medical informatics has evolved as a new field. Today, there are multiple challenges faced in medicine pertaining to record keeping like lots of paper-based records, medication errors and documenting patient related information. It deals with the resources, devices and methods required to optimize the acquisition, storage, retrieval and use of information in health and biomedicine. Health informatics tools include not only computers but also medical and health data, clinical guidelines, formal medical terminologies, knowledge for scientific inquiry and problem solving and decision making through information and communication systems. Health informatics technology includes the electronic information technology used during the course to track patient care and drawing insights from informatics data are of increasing value.

Healthcare and medical industry is undergoing a paradigm shift with plethora of big data in healthcare research and advances of data science in the medical domain. With the advancements of computer knowledge, internet and voice recognition and development of wearable health monitoring devices and wireless communication network, it is quite easy to collect complex volume of health data. Another powerful impetus which help in collection of large volume of data, previously inaccessible, is social media and health awareness thorough these platforms. Digital health monitoring devices are helping and playing an important role in educating people to identify telltale signs of serious medical conditions. As the technology will continue evolving and the information collected will pave path for new ideas, thinking and transformation of the overall healthcare industry and motivated to improve human health.

There are several medical technologies under development which has the potential to revolutionize the healthcare industry. Some of these medical technologies are orthopedic devices embedded with chips, screening device for cancer, and the Tricorder that can scan patients and help diagnose what is wrong. Due to the availability of such huge information of data and their availability through various applications, people are nowadays taking control of their own health in a highly personalized manner. These technologies are not only being transforming the care of chronic ill patients but also for those people who remain want to be healthy.

Integrating the latest developments in technology into the field of medicine is challenging and would require more healthcare professionals to become formally trained in technology. As the technology for collecting, analyzing and transmitting data in medical and health informatics continues to grow, there is possibility of more and more healthcare applications and systems to emerge. It will also need an additional education/training to understand the new systems prior to implementation. The need of faster microprocessor speed, better data storage, better bandwidth, use of artificial intelligence, cloud computing and more accurate sensors are the needs for future to better integrate with the patients portals and national information networks. These technologies will have broad reach, cost effective and mobile to people. The Journal of Health \& Medical Informatics invites and encourages scientists and researchers across the globe to explore the future of an expanding and shifting healthcare industry that will kindle the global efforts for reshaping the healthcare system.
*Corresponding author: Ritesh Kumar, Department of Bioinformatics, University of Kansas, Lawrence, KS, USA, Tel: +1-785-331-5356; E-mail: ritesh1612@gmail.com

Received August 10, 2017; Accepted August 21, 2017; Published August 25, 2017

Citation: Kumar R (2017) Paradigm Shift: Role of Social Media and Information Technology in Healthcare Advancements. J Health Med Informat 8: e146. doi: 10.4172/2157-7420.1000e146

Copyright: ( $) 2017$ Kumar R. This is an open-access article distributed under the terms of the Creative Commons Attribution License, which permits unrestricted use, distribution, and reproduction in any medium, provided the original author and source are credited. 